\title{
A Micromachined Airflow Sensor Based on RF Evanescent-Mode Cavity Resonator
}

\author{
Yanzhu Zhao, Seong-Hyok Kim, Yuan Li, Bo Pan, Xiaosong Wu, Manos Tentzeris, John Parapolymerou, and \\ Mark G Allen
}

School of Electrical and Computer Engineering, Georgia Institute of Technology

Atlanta, GA 30332

\begin{abstract}
This paper presents an RF airflow sensor based on an evanescent-mode cavity resonator. The frequency loading is achieved by a capacitive post in the center of a cylinder cavity. As a sensing mechanism, an elastic membrane with a sensing beam is utilized to generate a strain on the top of the cavity. The airflow deflects the sensing beam, changing the parasitic capacitance of the cavity and hence, the resonant frequency of the resonator. The cavity resonator is fabricated by micro-molding of thermal-plastic polymers enabling a low cost batch fabrication. The coplanar waveguide (CPW) feeding structure is formed simultaneously during the molding process by using a metal transfer technique. The resonant frequency of the resonator as a function of the sensing beam deflection angle is demonstrated, and a wind tunnel test is performed to demonstrate an equivalent sensitivity of $0.36 \mathrm{GHz} /(\mathrm{m} / \mathrm{s})$ for the air flow velocity measurement.
\end{abstract}

Index Terms - evanescent-mode, cavity resonators, airflow, CPW, micro-molding.

\section{INTRODUCTION}

Flow measurement is a necessary task in diverse fields as dynamic aircraft maneuvering, medical instrumentation, environmental monitoring, automotive applications, and process control. Many previous studies have demonstrated the successful application of micro-electro-mechanicalsystem (MEMS) techniques to the fabrication of a variety of flow sensors, capable of detecting both the flow rate and the flow direction [1-3]. However, few researchers report such flow sensors with the wireless capability that is essential for distributed sensor networks. Recently, RF cavity resonators have been utilized for sensor development [4-5]. Evanescent-mode cavity resonators better suit the need for sensor applications, because the size of the evanescentmode resonator is reduced and the sensitivity to the distance between the capacitive post and the top of the cavity becomes more pronounced [6-7]. A MEMS thermal sensor based on evanescent-mode cavity resonators fabricated by stereolithography has been demonstrated recently [8]. The batch fabrication of these air-lifted RF components such as air cavity or waveguide, as well as the feeding structures are usually achieved by surface micromachining or silicon DRIE $[9,10]$. However, it is challenging to integrate the sensing element on the top of the cavity due to the complexity of the fabrication. In addition, the feeding structures to excite the resonance are often on the top of the cavity, leaving limited space for the integration of sensing elements.

This paper proposes a novel airflow sensor based on an evanescent-mode cavity resonator. A CPW feeding structure on the bottom of the cavity is utilized to excite the cavity resonance. The simplicity of the feeding structures enables easy integration of the sensing elements. Metal-transfer molding (MTM) process-based MEMS technology is adopted for the realization of the cavity structures to achieve a low-cost batch fabrication [11-12]. The sensing element is fabricated separately and integrated with the cavity resonator by a simple fixture method.

\section{DESIGN}

Fig.1 shows the operation mechanism of the airflow sensor. An elastic membrane seals the cavity, and a miniature sensing beam is bonded on the membrane surface. The beam is deflected by the air flow, changing the resonance frequency of the highly loaded cavity resonator.

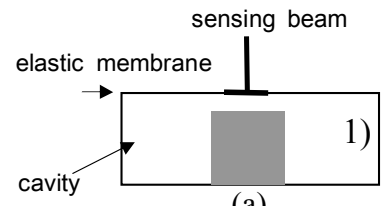

(a)

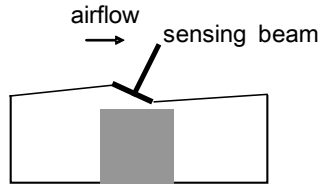

(b)
Fig. 1 The mechanism of the evanescent-mode cavity resonator airflow sensor (a) in static (b) in operation

Fig. 2 shows the design schematic of a $2 \times 2$ airflow sensor array based on an evanescent-mode cavity resonator. The sensor is assembled in three main parts including the cavity, membrane, and miniature sensing beam. Elastomer polydimethylsiloxane (PDMS) is utilized for the membrane and is coated with a thin layer $(3 \mu \mathrm{m})$ of copper for electrical functionality.

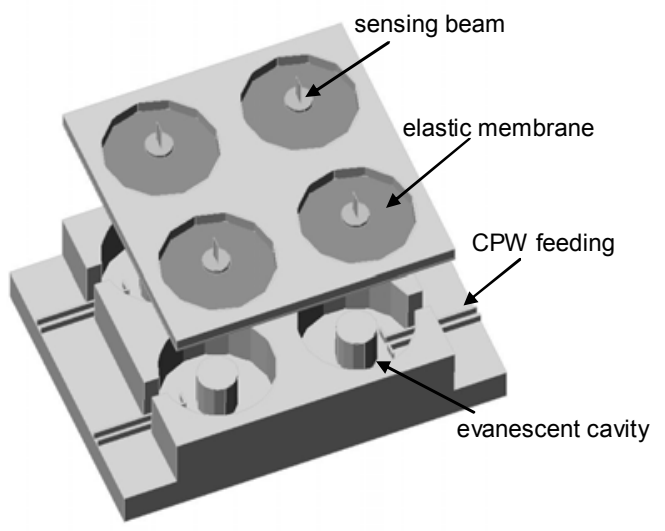

Fig. 2 Design schematic of the airflow sensor array 
Fig.3 shows the proposed evanescent-mode cavity resonator design which is composed of the cavity resonator and integrated CPW feeding line. The CPW feeding line critically couples the cavity resonator. The diameter of the designed cavity sample is $5 \mathrm{~mm}$ with a height of $1 \mathrm{~mm}$. The capacitive post diameter is $2 \mathrm{~mm}$ with a height of $0.85 \mathrm{~mm}$. The width of the CPW center conductor is $0.18 \mathrm{~mm}$ and the slot between the signal and the ground is $0.05 \mathrm{~mm}$. The insertion end of the CPW has a split slot structure with an arm of $0.9 \mathrm{~mm}$ at each end, as shown in Fig.3. HFSS fullwave analysis shows that at a certain width of the CPW split-end structure, a critical coupling from the CPW to the evanescent-mode cavity resonator can be achieved, and the S11 has a deep notch at the resonant frequency of the evanescent-mode cavity resonator, indicating a strong resonance.

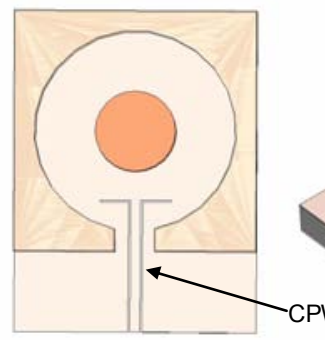

(a)

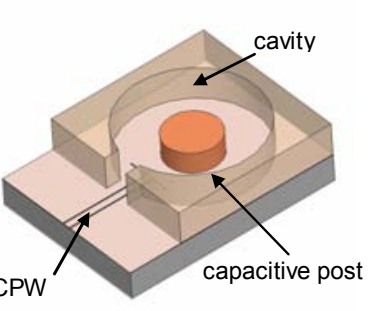

(b)
Fig. 3 Schematic of the evanescent-mode cavity resonator (a) top view (b) lateral view

Fig. 4 shows the simulated and measured resonance of this excitation. The tested cavity has a diameter of $5 \mathrm{~mm}$ and height of $2.5 \mathrm{~mm}$, and the capacitive post has a diameter of $2 \mathrm{~mm}$ and height of around $2.4 \mathrm{~mm}$. The simulated $\mathrm{S} 11$ has a deep return loss larger than $12 \mathrm{~dB}$ at a frequency of $10.35 \mathrm{GHz}$, which is close to the resonant frequency of the evanescent-mode cavity of $10.4 \mathrm{GHz}$, and the measurement shows good agreement with the simulation.

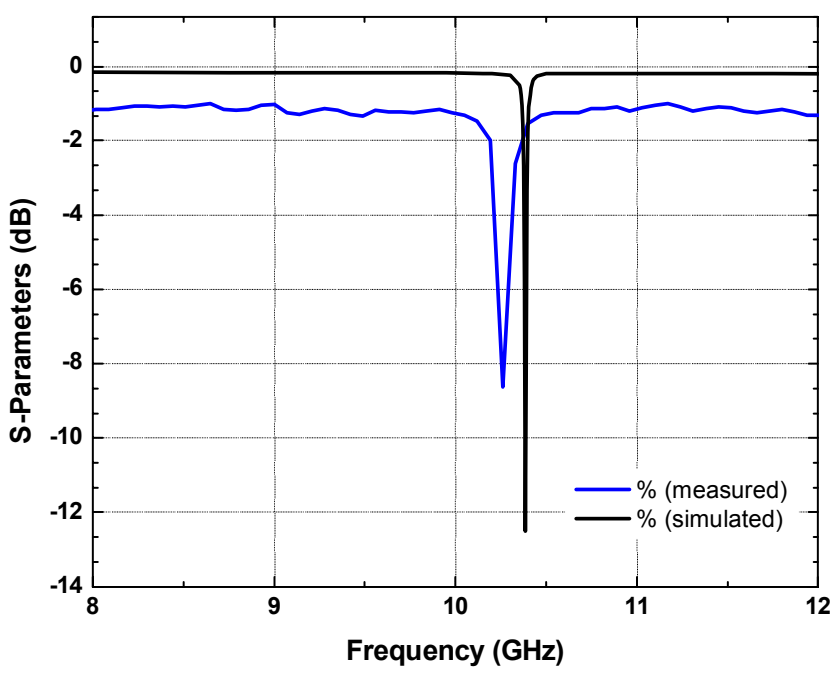

Fig. 4 Simulated and measurement of a CPW fed evanescent-mode cavity resonator

\section{FABRICATION}

Micromolding has emerged as a promising fabrication technology for microstructures not only due to its low-cost manufacturing potential, but also because it enables a wide choice of materials with desirable dielectric, mechanical and thermal properties. In this paper, micromolding technology is chosen as a low-cost and batch process to make the cavity resonators. The master for molding is made by stereolithography (SLA) and can be repetitively used for continuous molding processes. An MTM process is utilized to form the metalized 3-D structures as well as the CPW feeding lines [11].

Fig. 5(a) shows the fabricated cavity resonator array using this MTM process. The CPW feeding structures are inserted into the cavity through a small opening on the side of the cavity. Fig.5(b) shows the assembled sensor, which was developed using a plastic fixture. A $20 \mu \mathrm{m}$ thick elastic membrane is fabricated by spin coating PDMS solution, followed by thermal curing. The elastic nature of the membrane enables a high-strain-stress ratio. A thin copper layer is formed by sputtering on the membrane surface and reinforced by electroplating. The membrane is bonded and fixed on the top of the cavity by a fixture. A $3 \mathrm{~mm}$ long plastic beam is the bonded on the top of the cavity to facilitate measurement and can be integrated monolithically.

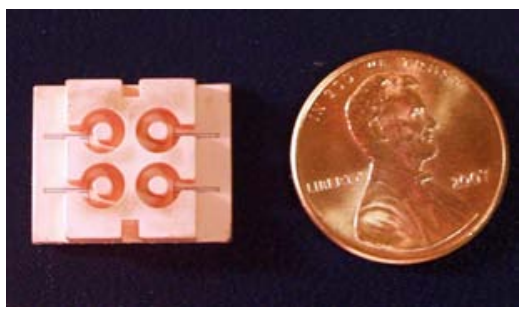

(a)

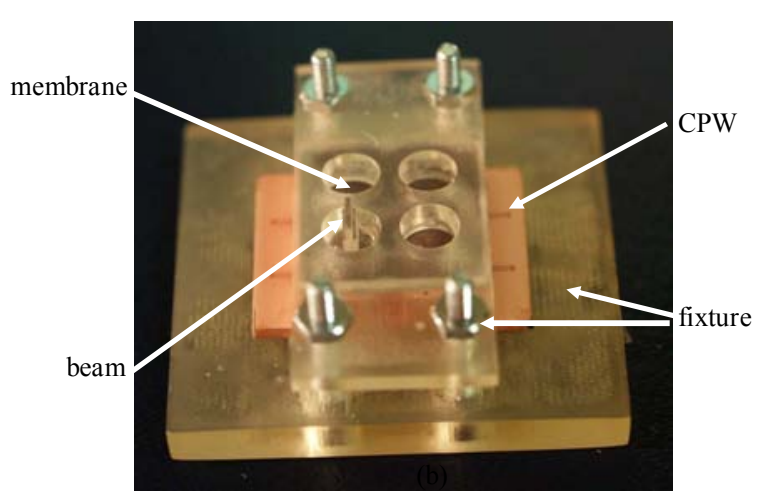

(b)

Fig.5 Fabricated airflow sensor array (a) fabricated evanescentmode cavity resonator array by MTM process (b) the assembled airflow sensor array by fixture

\section{MEASUREMENT AND ANALYSIS}

An RF measurement is performed first to characterize the RF properties of the airflow sensor using an Agilent $8510 \mathrm{C}$ network analyzer, in conjunction with an on-wafer 
probing station. Fig. 6 shows the experimental setup. The beam is deflected by gently applying a force, and both the deflecting angle and the resonant frequency are recorded at the same time.

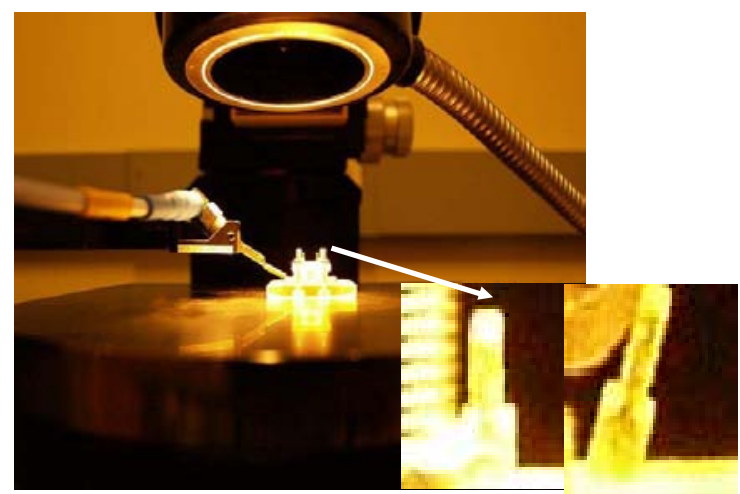

Fig.6 Measurement setup for the beam deflection and resonant frequency

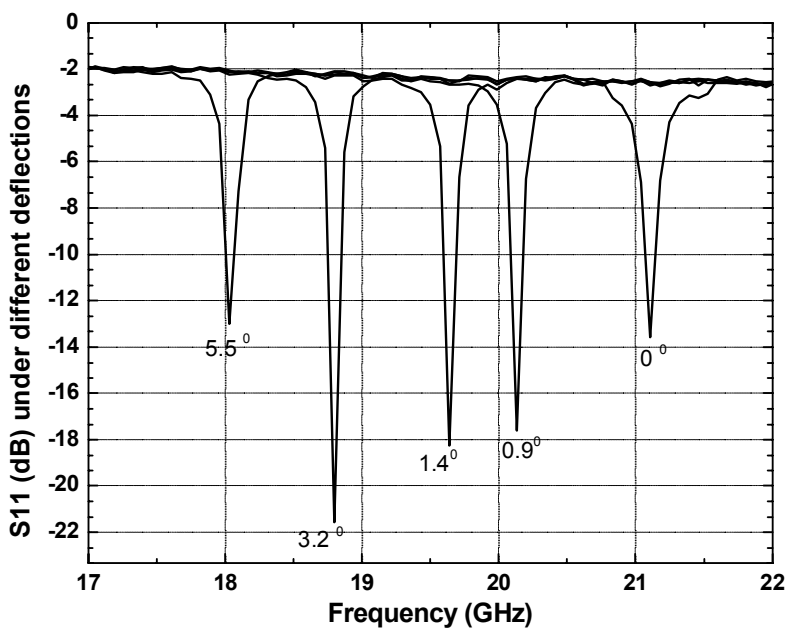

(a)

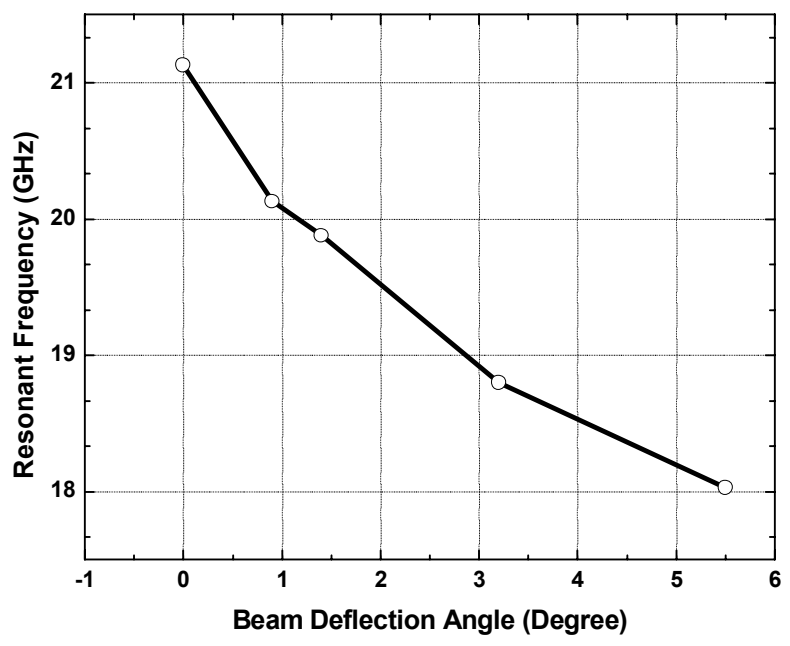

(b)

Fig.7 RF measurement results of the airflow sensor (a) measured S11curves at different deflection angles (b) measured resonance frequency shift versus the beam deflection angles
Fig. 7(a) shows the measured S11 at different deflection angles. When the beam is deflected, the effective capacitance increases, and the resonant frequency shifts lower, and a return loss of more than $10 \mathrm{~dB}$ is observed at the resonance. The sharp frequency selectivity is due to the high-Q nature of the microwave cavity resonator. Fig. 7(b) shows the measured resonant frequencies shift with the beam deflection. Approximately maximum $1.0 \mathrm{GHz} /$ degree change is observed from the measurement results, indicating a very high sensitivity achieved by the proposed sensor. The dynamic range of the sensor is decided by the maximum deflection angle when the top membrane touches the capacitive post inside the cavity.

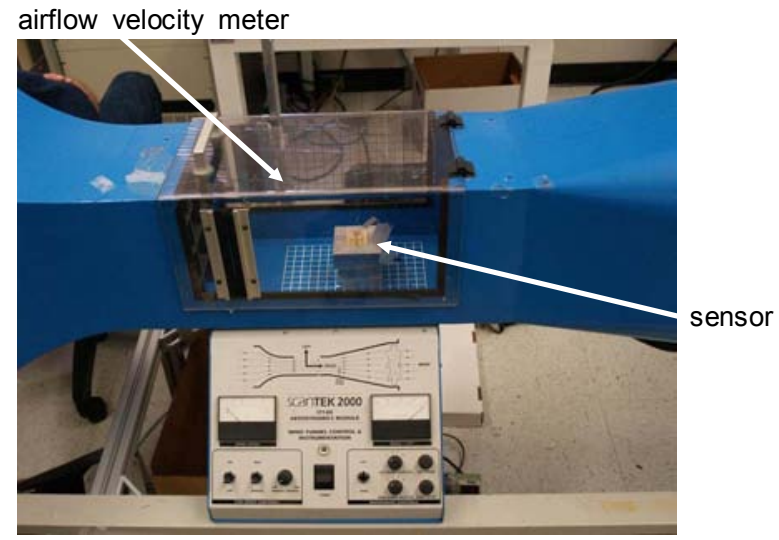

(a)

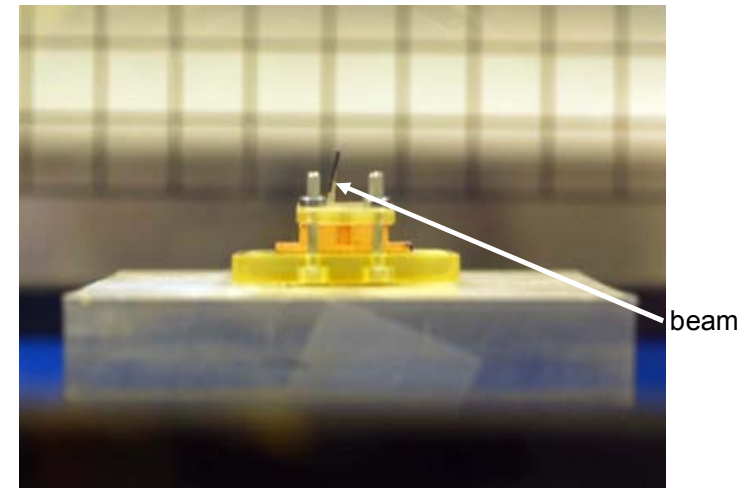

(b)

Fig. 8 Wind tunnel measurement of the evanescent-mode cavity based airflow sensor (a) wind tunnel setup (b) deflection of the sensing beam due to the airflow

Knowledge of the force-deformation characteristics of the resonant cavity allows the transduction of flow forces to readout frequency. The sensor is then put in the wind tunnel to characterize the airflow response. Fig. 8 shows the setup of the wind tunnel (ScanTEK2000). By adjusting the airflow velocity, the beam deflection angle is recorded. The airflow velocity is measured at the same time using a flow velocity meter.

Fig. 9 shows the measured results in the wind tunnel. The deflection angle has an approximately linear relation to the airflow velocity when the velocity is below $7.8 \mathrm{~m} / \mathrm{s}$. The nonlinear relation beyond $7.8 \mathrm{~m} / \mathrm{s}$ is mostly due to the contact of the top membrane with the capacitive post inside the cavity. An approximately $1.4 \mathrm{~m} / \mathrm{s}$ per degree change of the beam angle can be observed in the linear region, and 
according to the RF characteristic of the sensor, an equivalent sensitivity of more than $0.36 \mathrm{GHz} /(\mathrm{m} / \mathrm{s})$ has been achieved for the airflow velocity sensing by the evanescent cavity resonator based sensor. This can be easily captured during an RF measurement.

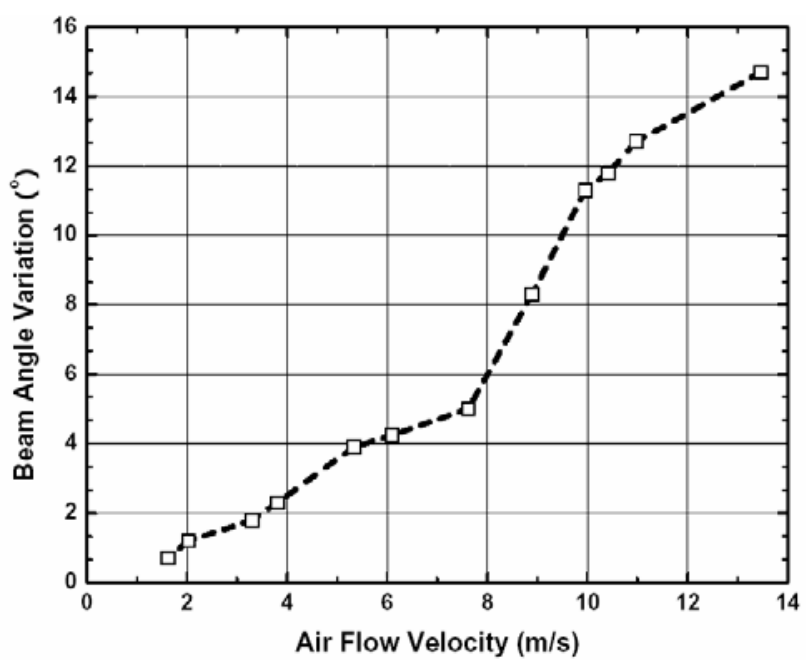

Fig. 8 The measured deflection angle versus airflow velocity

\section{CONCLUSION}

An RF airflow sensor based on evanescent-mode cavity resonator was demonstrated. Miniature sensing beam and thin elastic membrane were used to change the resonant frequency of a highly loaded evanescent-mode cavity resonator. The airflow sensor proposed in this paper has shown the change of the resonant frequencies from $21.1 \mathrm{GHz}$ to $18.0 \mathrm{GHz}$ by deflecting the sensing beam. This result indicates a frequency sensitivity of $0.36 \mathrm{GHz} /(\mathrm{m} / \mathrm{s})$ for the airflow velocity measurement.

\section{ACKNOWLEDGMENT}

This project is supported by the Department of Defense AFOSR MURI program, grant number FA9550-05-1-411.

\section{REFERENCES}

[1] Matova, S.P.; Makinwa, K.A.A.; Huijsing, J.H. "Compensation of packaging asymmetry in a 2-D wind sensor," IEEE Sensors Journal 2003, 3, 761-765.

[2] Mailly, F.; Giani, A.; Bonnot, R.; Temple-Boyer, P.; PascalDelannoy, F., Foucaran, A.; Boyer, A. "Anemometer with hot platinum thin film," Sensors and Actuators A 2001, 94, 32-38.

[3] Kim, S.; Nam, T.; Park, S. "Measurement of flow direction and velocity using a micromachined flow sensor," Sensors and Actuators A 2004, 114, 312-318

[4] M.G.Allen, "Micromachined Endovascularly-Implantable Wireless Aneurysm Pressure Sensor: From Concept to Clinic", Proc. Transducers 2005, Vol1, pp275-278

[5] J. Chuang, D.J. Thomson, and G.E.Bridges, "Embeddable wireless strain sensor based on resonant RF cavities", Review of Scientfic Instruments, 76, 094703, 2005
[6] X. Gong, A. Margomenos, B. Liu, W. J. Chappell, and L. P. B. Katehi, "High-Q evanescent-mode filters using silicon micromachining and polymer stereolithography (SL) processing," IEEE MTT-S Int. Microwave Symp. Dig., vol. 2, pp. 433-436,

[7] H.Joshi, H.H.Sigmarsson, D.Peroulis and W.J.Chappell, "Highly Loaded Evanescent Cavities for Widely Tunable High-Q Filters", IEEE MTT-S 2007

[8] A. Mahmood, H.H. Sigmarsson, H.Joshi, W.J.Chappell, and D.Peroulis, “An Evanescent-mode Cavity Resonator Based Thermal Sensor", Proc. of 2007 IEEE sensors, pp.950-953

[9] B.Pan, Y.Li, M.M.Tentzeris, J.Papapolymerou, "A High-Q Millimeter-Wave Air-Lifted Cavity Resonator on Lossy Substrates", IEEE Microwave and Wireless Components Letters, Vol.17, No.8 P571-573

[10] Y.Li Y. Li, B. Pan, M.M.Tentzeris and J. Papapolymerou, "A Fully Micromachined W-Band Coplanar Waveguide to Rectangular Waveguide Transition”, 2007 IEEE-IMS Symposium, pp.1031-1034

[11] Y.Zhao, Y.Yoon, and M.Allen, "Metal-Transfer-Micromolded RF Components for System-On-Package (SOP)", Proc. of 57th Electronic Components and Technology Conference, ECTC'07, pp.1877-1883

[12] Y.Zhao, Y.Yoon, X.Wu, and M. Allen, "Metal-TransferMicromolded Air-lifted RF Components", Transducers", Proc. of the 14th International Conference on Solid-State Sensors, Actuators and Microsystems, 2007, pp. 659-662 